\title{
Neutrino Background from Population III Stars
}

\author{
Fabio Iocco \\ Università di Napoli "Federico II", Napoli, Italy \\ KIPAC @ Stanford/SLAC, CA, USA \\ E-mail: iocco@na.infn.it
}

\begin{abstract}
Population III Stars (PopIII) are the first generation of stars formed from the collapse of the very first structures in the Universe. Their peculiar chemical composition (metalfree, resembling the Primordial Nucleosynthesis yields) affects their formation and evolution and makes them unusually big and hot stars. They are good candidates for the engines of Reionization of the Universe although their direct observation is extremely difficult. Here we summarize a study of their expected diffuse low-energy neutrino background flux at Earth.
\end{abstract}

PopIII are the pregalactic generation of stars which formed from the pristine metal-free gas left from Primordial Nucleosynthesis. The dust- and metal-free gas from which they originated made them unusually heavy $\left(\mathcal{O}\left(200 \mathrm{M}_{\odot}\right)\right)$, extremely short lived $\left(\mathcal{O}\left(10^{6}\right) \mathrm{yrs}\right)$, and with a high rate of Pair Instability Supernovae (PISNe). Their peculiarities make them extremely difficult to observe directly and so far no unambiguous observation has been reported. Such massive stars should have a high Supernova rate, and it is therefore reasonable to expect a huge $\nu$ emission associated with the PopIII phenomenon. We study their MeV-energy neutrino flux to understand if it can be considered as a direct observable for such a generation of stars, motivated also by the recent interest in modeling the diffuse fluxes due to different cosmic sources. Our simple model, which may be regarded as an upper limit to the actual flux, makes strong assumptions on the Stellar Formation Rate (SFR), the Initial Mass Function (IMF) of the PopIII and uses the results of 3-D simulations of PISNe explosions to model the $\nu$ contributions. In the model we propose and fully describe in [1] we assume that the whole PopIII generation is formed by only $300 M_{\odot}$ stars, which give the most spectacular contribution in neutrinos, $\approx 10^{55} \mathrm{ergs}$, according to [2]; we also assume that a fraction $f_{\mathrm{III}}=10^{-3}$ of the total baryonic mass of the Universe contributes to the stellar phenomenon (actually ends up in a star) which we model as a delta-like function at the redshift suggested from WMAP3 as the central value for Reionization, $\bar{z}=12$. The neutrino contributions we take into account are the $\nu_{e}$ flux due to H-burning $(E<2 \mathrm{MeV})$, the $\nu$ and $\bar{\nu}$ of all flavours by purely leptonic processes and $\nu$ and $\bar{\nu}$ of all flavours produced during the PISNe collapse $(E \leq 20 \mathrm{MeV})$, energies at the source. We find that, as expected, the energy at the Earth is lower because of cosmological redshift effect: the $\nu$ flux drops below $1 \mathrm{~cm}^{-2} \mathrm{~s}^{-1}$ at $E \approx 5 \mathrm{MeV}$, the total background being always dominated by cosmic $\mathrm{SNe}$ and galactic stellar neutrinos. Although the anti-neutrino flux shows the same magnitude it is interesting to notice that the PopIII flux dominates the cosmic background up to $E \approx 2 \mathrm{MeV}$.

\section{References}

[1] F. Iocco, G. Mangano, G. Miele, G.G. Raffelt and P.D. Serpico, Astropart. Phys. 23 (2005) 303.

[2] C.L. Fryer, S.E. Woosley and A. Heger, Astrophys. J. 550 (2001) 372. 